\title{
Extracellular volume fraction measured by MOLLI: slow infusion versus bolus
}

\author{
Erik Schelbert ${ }^{1 *}$, Stephen M Testa ${ }^{1}$, Christopher G Meier ${ }^{1}$, William J Ceyrolles ${ }^{1}$, Joshua Levenson ${ }^{1}$, Alex J Blair ${ }^{2}$, \\ Kathy S Puntil', Peter Kellman³, Bobby L Jones ${ }^{1}$, Daniel R Ludwig' ${ }^{1}$, Hua Zhong ${ }^{1}$, David Schwartzman', \\ Sanjeev G Shroff', Timothy C Wong ${ }^{1}$
}

From 2011 SCMR/Euro CMR Joint Scientific Sessions

Nice, France. 3-6 February 2011

\section{Background}

Myocardial extracellular volume fraction (Ve) measures from myocardial and blood pool T1 data quantify diffuse fibrosis (Flett, 2010) not detectable by conventional late gadolinium (Gd) enhancement, but require steady state equilibrium between plasma and interstitium. While a bolus with a lengthy infusion produces steady state for Ve measures, it is unclear whether a Gd contrast bolus alone (e.g., Broberg, 2010) is sufficient. Given the relatively slow clearance of $\mathrm{Gd}$, we hypothesized that a simple bolus accurately measures $\mathrm{Ve}$, thus facilitating integration of $\mathrm{Ve}$ measurement into CMR workflow routines.

\section{Methods}

In 10 diverse volunteers (ages 20-81, median 33 yr), we compared serial Ve measures from two scans: first, during a constant infusion $(0.1 \mathrm{mmol} / \mathrm{kg}$ bolus followed by $0.1 \mathrm{mmol} / \mathrm{kg}$ diluted in $200 \mathrm{~mL}$ saline, $200 \mathrm{~mL} / \mathrm{hr}$ infusion, $\mathrm{x} 1 \mathrm{hr})$, and second, $12-50 \mathrm{~min}$ after a bolus $(0.2 \mathrm{mmol} / \mathrm{kg})$ on another day. Ve data from a diastolic short axis slice were computed as described by Jerosch-Herold (2008). Steady state during infusion was defined when blood and myocardial $\mathrm{T} 1$ varied $<5 \%$. We measured $\mathrm{T} 1$ on a $1.5 \mathrm{~T}$ Siemens scanner using a single-shot modified Look Locker inversion recovery research sequence (MOLLI), similar to Messroghli (2007). Pre-contrast (longer) T1 values were measured with $2 \mathrm{RF}$ pulses and $5+1$ heart beat sampling scheme, and post-contrast (shorter) T1 measures used 3 RF pulses and $4+2+1$ sampling scheme with at least 1-2 dummy beats separating RF pulses from preceding SSFP readout. At fast or slow heart rates $(65-95 \mathrm{bpm})$ we validated this technique using CuSO4-Agar phantoms with

${ }^{1}$ University of Pittsburgh, Pittsburgh, PA, USA

Full list of author information is available at the end of the article similar T1 and T2 of myocardium/blood, pre- and postGd as measured by spin echo inversion recovery (T1) and saturation recovery (T2), $1 \mathrm{k}$-space line/RF pulse, $\mathrm{TR}=15 \mathrm{~s}$. MOLLI and spin echo T1 measures $(\mathrm{n}=210)$ correlated well (slopes $>0.95 ; \mathrm{p}=\mathrm{NS}$ vs. unity) without bias on Bland-Altman plots. Infusion vs. bolus Ve measures $(n=205)$ across subjects were compared with generalized estimating equations with exchangeable correlation matrices for serial measures.

\section{Results}

By infusion, the Ve range was 19.3-29.2\% with a $\mathrm{SD}<=0.9 \%$ for repeated measures $(\mathrm{n}=110)$ in the 10 subjects. By bolus, the Ve range was $17.4-29.1 \%$, with a $\mathrm{SD}<=1.3 \%$ for the repeated measures $(\mathrm{n}=95)$. Serial Ve measures by bolus or infusion did not differ significantly (delta $=0.2 \%, \mathrm{p}=0.20)$.

\section{Conclusion}

Serial Ve measures from either an intravenous bolus or infusion did not differ significantly in a diverse sample of volunteers. Myocardial Ve can be measured reliably and accurately $12-50$ minutes after a simple $0.2 \mathrm{mmol} / \mathrm{kg}$ bolus.

\footnotetext{
Author details

${ }^{1}$ University of Pittsburgh, Pittsburgh, PA, USA. ${ }^{2}$ Carnegie Mellon University, Pittsburgh, PA, USA. ${ }^{3}$ National Heart, Lung, Blood Institute, Bethesda, MD, USA.

Published: 2 February 2011

doi:10.1186/1532-429X-13-S1-P317

Cite this article as: Schelbert et al:: Extracellular volume fraction

measured by MOLLI: slow infusion versus bolus. Journal of Cardiovascular

Magnetic Resonance 2011 13(Suppl 1):P317.
} 